\title{
Survey: Problems Related to Human in Software Projects
}

\author{
${ }^{1}$ Mohammed A. Othman, ${ }^{2}$ Sherif A. Mazen, ${ }^{3}$ Ehab Ezzat \\ ${ }^{I}$ Faculty of Computers and Information, Cairo University, Cairo, EGYPT \\ ${ }^{2}$ Assoc Prof., Information Systems Department, Cairo University \\ ${ }^{3}$ Assoc. Prof., Information Systems Department, Cairo University
}

\begin{abstract}
The human factor is the most significant success factor of any project, in this paper we will explain some important problems related to managing human factor in software projects; and we will survey on all important problems that face projects managers and affect project outcomes. This survey will help to concentrate on the most important problems and Proceed to introduce guides and solutions to overcome these problems. this paper is the starting point toward develop a new framework for managing technical people in software projects, this research will cover the most significant problems which arise during projects management specifically in software projects in technical team; these problems almost lead to projects failure, or delivered out of budget and/or planned time, some of these problems related to team selection, team evaluation, time management, workload distribution, poor planning, and tasks allocations and distribution, in this survey we will explain and introduce the most important studies, researches, publications, and ideas related to team management in software development.
\end{abstract}

Keywords: Software Project Management, Technical Team Management (TTM), Software Projects (SWP).

\section{Introduction}

Technical team management is the very important part of projects management is SW projects [4]. Your team is the most important resource you have available and their enthusiastic contribution will make or break your project [6]. In this research we will survey, and study how to enhance the technical team management in software projects in order to lead the project to success and delivered in time and planned budget. The human is the most significant success factor in any project, so the researches and studies in the management of human resources in software project become a hot research area and important matter, also the published studies $[12,13]$ in people management in software projects show that the technical team can be one from the most causes of projects failure. A project is a temporary endeavor with a defined beginning and end (usually time-constrained and often constrained by funding or deliverables) [2]. Projects Management is an Organizing, securing managing, leading, and controlling resources to achieve specific goals. Software project management is the art and science of planning and leading software projects [1]. Software projects management: it's a sub discipline of project management in which software projects are planned, monitories, and controlled. The software development methodologies, approaches, researches, and published studies till now almost interested in the software project as a final product, even in the development phases discussed only the steps of analysis, design, testing, of the software project itself.

\section{People In Software Projects}

Information Technology (IT) project Management (software project management) is a sector that has arguably witnessed the highest rate of project failure [4]. People are an organization's most important assets, the tasks of a manager are essentially to be people-oriented, unless there is some understanding of people, and management will be unsuccessful [3]. Poor people management is an important contributor to project failure [3]. According the graph below which show the people is the most important factor in software development process 


\section{Software Quality Determinants [4]}

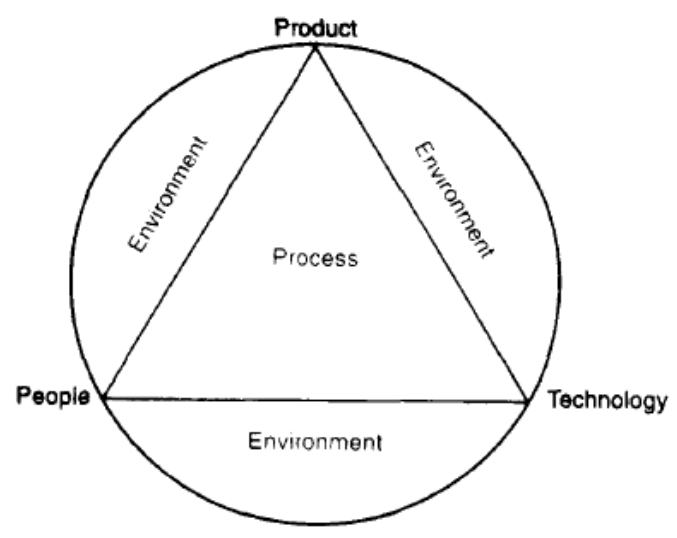

III. Related Work

Some methodologies and approaches interested in human factor in software development process like Agile Methodology, Rapid Application Development RAD; but there is no a complete study and guides discuss and study all human aspects, specifically in software projects, although some studies appear to disuses only some aspects of human factor [12]. People-related issues are central factors in determining the success of software projects; indeed, many software projects fail in terms of meeting customer needs, on-time delivery, and budget. Since the importance of the human aspects of software engineering (SE) to the success of software projects has already been acknowledged, why do these aspects not receive more attention in the software industry in order to improve project Results?[12].

\subsection{Planning problem}

\section{Main Problems}

Sometimes IT managers are not given the opportunity to plan because time pressure from senior management take over and most of the time the project is on its way before it has been clearly defined [6]. Detailed plans are not effective for managing IT work. The reason is that the managers do not know enough about the work to make detailed plans [11]. Team members might make their own plan but most of them do not want to. They would rather implement the solution. Few of them have the skills and experience to make complete plans, and there is a big risk in trusting them in producing their own plans that will meet management objectives [11].

\subsection{Unclear goals and objectives for the project technical teams:}

Sometimes the goal of a project may be only partially clear due to a poor requirement gathering in the definition stage of a project [7]. This affect on the team work performance because they work in unclear area, they can't plan a blind objectives.

\subsection{Poor estimation of team size}

Assume we have a large IT project using a team with a staff of one hundred people. Linear thinking would support the conclusion that increasing the people by 100 percent would decrease the schedule and increase the cost to approximately the same degree. In reality, doubling the staff produce a non-linear result [9]. The team size must be determined based on some factors, size of project, type of technology used, knowledge level and culture of surrounding environment, and time project.

\subsection{Lack of executive support and user involvement:}

The research companies and academic institution has focused on the lack of executive support and user involvement as two main difficulties in managing IT projects [10]. The user involvement is very important in any project because the involvement instill a sense of affiliation and responsibility.

\subsection{Communication problems}

Failure to communicate and act as a team Projects sometimes fail due to improper communication. Science Application International Corporation said that the communication with FBI was difficult because of the high turnover of top IT managers [9]. Communication problems are common on large IT projects. Because complex IT projects often involve large amount of analysis and work, the project teams are busy and the 
executive management sees no progress. IT project managers do not communicate progress regularly because they believe that progress will not be seen by the executive management [7]. The better communication between team members, the better of project results, deliverables, and outcomes.

\subsection{Inappropriate skills:}

The challenge of global competition, the rapid growth of knowledge, and the constant changes of technology make it hard to predict what kind of skilled people will be needed.

Most IT projects require a diverse range of skills. Many teams lack the breadth, and depth they require [8]. It is also not easy for technology based organization to find the experienced people they need because sometimes few people in the labor market have the necessary skills.

The larger the project, the more need there is also for people with excellent planning, oversight, organization, and communications skills; experienced technology skilled people do not necessarily have these abilities [7]. We know the famous "CHAOS summary 2009" reports pointed out that $44 \%$ of software projects were delayed, and $24 \%$ of software projects were failed [5]. If we have evaluate the team members before selection, task assignment, and hunt the high skilled persons; that lead to decrease the problems of skills availability.

\subsection{Objectives changing during the project:}

Scope creep refers to uncontrolled and unexpected changes in user expectations and requirements as a project progress, while feature creep refers to uncontrolled addition of features to a system with a wrong assumption that one small feature will add nothing to cost or schedule [8]. Unexpected changes in requirements absolutely affect on team performance specifically if the new requirements need unavailable skills in team members, that's will affect on time of project, training, or outsourcing budget.

\subsection{Lake of Management Skills in Team Members}

Human-related issues are central factors in determining the success of software projects [12]; indeed, many software projects fail in terms of meeting customer needs, on-time delivery, and budget. Since the importance of the human aspects of software engineering (SE) to the success of software projects has already been acknowledged, why do these aspects not receive more attention in the software industry in order to improve project results [12]? We suggest putting a human aspects and management skills in the academic Curclam of software engineering and computer since departments.

\subsection{Team Evaluation}

The traditional methods of team member's evaluation almost done during the project execution progress, and after tasks time we need to concentrate on team member evaluation before tasks allocated to the team members, a proposed evaluation method needed to used at the beginning of project lifecycle; if the right evaluation done before team selection this will guarantee the skilled people will selected. Also the evaluation of performance during the project life cycle must be done normally.

\subsection{Resources Allocation}

Planning a large scale software project involves a set of activities and an allocation of members to teams and teams to work packages. Human resource is the most important asset for a company to be competitive. Given a fixed Work package ordering, there exists an optimal people distribution into teams. As the complexity of projects increases, the requirement of an organized planning and scheduling process is enhanced [4]

The problem of resource allocation can be overcome by do a suitable profile much up between what we have, and what we want to do; and make resources to deliverable mapping, and allocation.

\section{Conclusion}

In this paper we tried to cover the most related problems in people management in software projects, because most of the development methodologies and approaches interested only in the software product as final unit and not deeply analysis the causes of poor management of technical people which may be cause a project failure or delay, we have introduce this survey to cover all important problems, and this survey will act as a starting point to solve these problems. We noted that only some studies discuses different aspects of people management in software projects, like agile software development methodology which interested in team work and force that team must be well organized and good communication strategies follow. Also Rapid Application Development RAD interested in people and team members' involvement, also some frameworks developed to manage the software projects like SCRUM framework which say that the team must be self organized and introduce a new way to manage team work. 


\section{Future Works}

During this research we noted reached to some points that needs to make a complete study and add a new procedures and techniques to enhance and improve the management of technical people in software projects, this points like tasks allocation and distribution, team members Evaluation, and selection criteria based on some standards and certifications, also we need to develop a maturity model for people skills and competencies evaluation and measurements, also we need to study what about the technical team management if all companies migrate its software to cloud computing

\section{References}

[1] Bradley L. Kirkman, Kevin B. Lowe, and Dianne P. Young "High-Performance Work Organzations", Center for Creative Leadershi $\odot 1999$

[2] B.N. Singh, Bhim Singh, Ambrish Chandra, and Kamal Al-Haddad, "Digital Implementation of an Advanced Static VAR Compensator for Voltage Profile Improvement, Power Factor Correction and Balancing of Unbalanced Reactive Loads", Electric Power Energy Research, Vol. 54, No. 2, 2000, pp. 101-111.

[3] Ian Somerville, "Software Engineering $7^{\text {th }}$ Edition, Ch.25.

[4] Daniel J. Paulish, Anita D. Carleton, "Case Studies of Software-process-improvement Measurement" IEEE, Volume 27 Issue 9, September 1994 Page 50-57.

[5] Nianfang Ji, Jie Wang" A Software Project Management Simulation Model Based on Team Climate Factors Measurement", IEEE 0018 - 9162/94 1994.

[6] New Zealand Management, "When IT Projects Fail", Volume 50, Issue 2, page 18. Available from Business Source Premier Database at http://www.ebscohost.com [Accessed Dec 25, 2011]

[7] Glaser, J "Management's role in IT project failures" Healthcare Financial Management, October, 2004.

[8] Fichter, Darlene "Why Web Projects Fail "[Online Journal] Online, Volume 27, Issue 4, page 43. Available from computer source database at http://www.ebscohost.com [Accessed July 25, 2011], 2003.

[9] Gross, Grant "FBI trying to salvage \$170 million software package [Online] IDG News Service. Available from: http://www.computerworld.com/printthis/2005/0 ,4814,98980,00.html [Accessed July 31, 2011]

[10] Jenster, P and Hussey,"Create a common culture between IT and business people to reduce project failures "Computer Weekly, March 22, 2005.

[11] Humphrey, "Why Big Software Project Fail: The 12 Key Questions" The Journal of Defense Software Engineering, March Issue, 2005

[12] Orit Hazzan, “ Putting Human Aspects of Software Engineering in UniversityCurricula”, Philippe Kruchten University, 07407459/1 IEEE, 2010

[13] Javier Garcı'a Guzma'n • Javier Saldaña Ramos • Antonio Amescua Seco • Ana Sanz Esteban, "How to get mature global virtual teams: a framework to improve team process management in distributed software teams", Springer Science+Business Media, LLC $2010,8: 409-435$. 